\title{
POINT ARBORICITY CRITICAL GRAPHS EXIST
}

\section{BÉLA BOLLOBÁS AND FRANK HARARY†}

\begin{abstract}
The point arboricity of a graph is the minimum number of colours assignable to the points so that no cycle is monochromatic. A graph is called $k$-critical if it is connected and the removal of any edge reduces the point arboricity from $k$ to $k-1$. The existence of $k$-critical graphs of odd order only was established by Kronk and Mitchem. We construct here $k$-critical graphs of every possible even order.
\end{abstract}

\section{Introduction}

In general, we follow the notation and terminology of the book [2], but we use the terms point and edge. The order of a graph $G$ is the number $p$ of its points. The symbol $G \cup H$ will always refer to the union of disjoint graphs.

We write $n G$ for the union of $n$ disjoint copies of a connected graph $G$. Also $G_{1}+G_{2}$ is the join of $G_{1}$ and $G_{2}$, consisting of $G_{1} \cup G_{2}$ and all edges joining (a point of) $G_{1}$ with $G_{2}$. As usual $\bar{H}$ denotes the complement of a graph $H$ and $G-e$ is the subgraph of $G$ resulting from the removal of the edge $e$. The addition of a new edge $e$ joining two non-adjacent points of $G$ results in the graph $G+e$.

The subject of the (edge) arboricity of a graph was launched by Nash-Williams [4]. He obtained an exact determination of the arboricity of a graph in terms of its subgraphs, see [2; p. 90].

A colouring of the points of a graph is called acyclic if no cycle has all its points the same colour. The point arboricity $\rho(G)$ of a graph $G$ was introduced by Chartrand, Kronk and Wall [1] as the minimum number of colours in an acyclic colouring of the points of $G$. A graph $G$ is $k$-critical with respect to point arboricity if $G$ is connected, $\rho(G)=k$ and for each edge $e, \rho(G-e)=k-1$. Obviously the 2-critical graphs are the cycles, so henceforth we take $k \geqslant 3$.

Kronk and Mitchem [3] established that for each $k \geqslant 3$ and odd $p \geqslant 2 k-1$, there exists a $k$-critical graph of order $p$. This was done by verifying that the graph $G=K_{2 k-4}+C_{p-2 k+4}$ has this property. They could not decide whether or not there exists a $k$-critical graph of even order but they showed that for $k \geqslant 3$ there is no $k$-critical graph of order $2 k$ or of order $p \leqslant 2 k-2$. Our object is to prove that there exist $k$-critical graphs of each even order greater than $2 k$. This is done by constructing appropriate families of $k$-critical graphs.

\section{Preliminary results}

The following lemmas are needed in the proof of our main results. As Lemma 1 is an elementary observation, we state it without proof.

LEMMA 1. If a connected graph $G$ does not contain three independent points, then every induced subgraph of order 5 contains a cycle.

Received 25 April 1974; revised 23 October 1974.

$\uparrow$ Research supported in part by Grant 73-2302 from the Air Force Office of Scientific Research.

[J. LoNDON Math. Soc. (2), 12 (1975), 97-102] 
LEMMA 2. For each $h \geqslant 1$, there is a hamiltonian triangle-free graph $\mathrm{H}$ of order $4 h+3$ such that the addition of any new edge e results in a graph $H+e$ which contains $a$ spanning subgraph of the form $K_{3} \cup 2 h K_{2}$, the union of a triangle with $2 h$ disjoint edges.

Proof. If $h=1$, it is easy to check that the required graph $H$ may be obtained from $C_{7}$ by adding any three disjoint diagonals, which do not produce a triangle (Fig. 1).

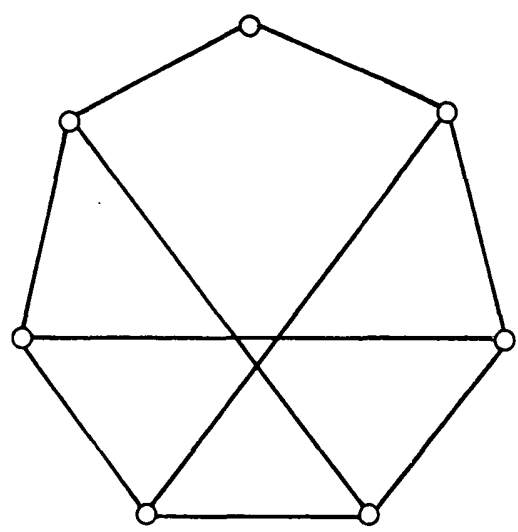

Fig. 1. A graph $H$ for $h=1$.

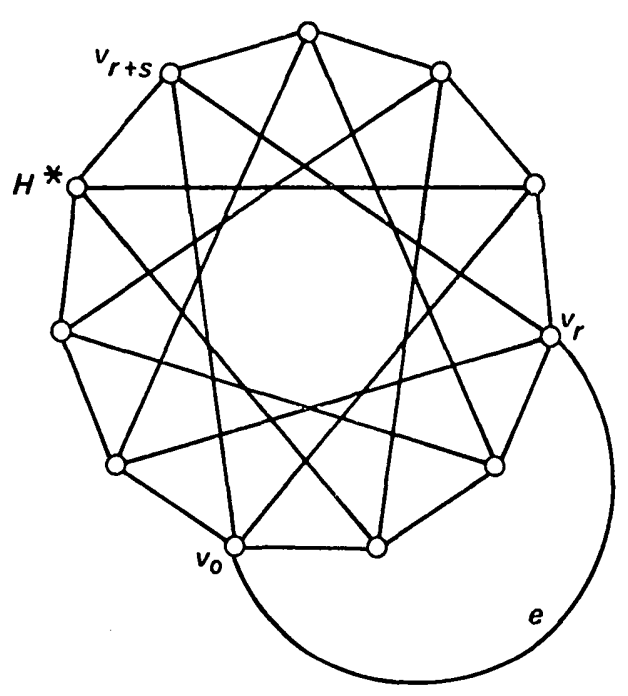

Fig. 2. A graph $H^{*}$ for $h=2$.

Suppose now that $h \geqslant 2$. In order to construct an appropriate graph $H$, start with the graph $C=C_{4 h+3}$ and join every pair of points at distance $2 h$ in $C$. Call these new edges the chords of $C$. The resulting graph has no triangles. Add edges arbitrarily until a maximal triangle-free graph $H$ is obtained. Obviously $H$ is hamiltonian and triangle-free, and has order $4 h+3$.

We shall show that $H$ satisfies the condition of Lemma 2. Let $e$ be any edge of $\bar{H}$ and put $H^{*}=H+e$. Then $H^{*}$ contains a triangle $K$ (see Fig. 2). To prove the lemma we show that the subgraph $H^{*}-V(K)$ has a 1 -factor, i.e.,

$$
H^{*}-V(K) \supset 2 h K_{2} .
$$

Label the points of $C$ by $v_{0}\left(=v_{4 h+3}\right), v_{1}, \ldots, v_{4 h+2}$, so that $v_{i}$ is adjacent to $v_{i+1}$, $i=0, \ldots, 4 h+2$. Without loss of generality, let $V(K)=\left\{v_{0}, v_{r}, v_{r+s}\right\}$, where

$$
0<r \leqslant s \leqslant t=4 h+3-(r+s) .
$$

If $r$ and $s$ are odd then so is $t$. As $C-V(K)$ is the union of disjoint paths with $r-1, s-1$ and $t-1$ points, the alternating edges from these paths form the required 1-factor.

Thus we can suppose that exactly one of $r, s$ and $t$ is odd and $t w o$ are even.

We now describe how to find a chord $x$ independent of the edges in $K$ such that $C-V(K)-V(x)$ is the union of disjoint paths of odd length. The edge $x$ together with alternating edges from these paths will form the desired 1-factor.

If $r$ is odd then choose the edge $v_{r+1} v_{r+1+2 h}$ for the chord $x$. As $s$ and $t$ are even 
and $r+s<r+1+2 h<4 h+3$, this chord has the required properties, since

and

$$
r-1, r+s-(r+2)=s-2, r+1+2 h-(r+s+1)=2 h-s
$$

are all even.

$$
4 h+3-(r+1+2 h+1)=2 h+1-r
$$

If $s$ is odd then we take $x$ to be the chord $v_{r-1} v_{r+2 h+2}$. As

$$
r+2<r+2 h+2<4 h+3,
$$

the appropriate paths contain $r-2, s-1,2 h+1-s$ and $2 h-r$ points.

Finally, if $t$ is odd then we can choose $x=v_{r+1} v_{r+2 h+4}$. As

$$
r+s<r+2 h+3<4 h+3
$$

$C-V(K)-V(x)$ is the disjoint union of four paths of even length, namely, of length $r-1, s-2,2 h+2-s$ and $2 h-1-r$.

\section{The Existence Theorem}

By the results of Kronk and Mitchem [3], the existence of a $k$-critical graph $(k \geqslant 3)$ of order $p$ has been established for all odd $p \geqslant 2 k-1$ and the impossibility has been shown for $p-2 k$ and $p<2 k-1$. Our theorem asserts the existence for all remaining even $p \geqslant 2 k+2$. The proof which we have been able to devise involves three separate propositions, whose combination yields the results: In Proposition 1, the existence of a $k$-critical graph of even order $p$ is proved for $2 k+2 \leqslant p \leqslant 3 k-3$; Proposition 2 shows it for $3 k-2 \leqslant p \leqslant 4 k-4$; Proposition 3 handles the remaining $p \geqslant 4 k-3$. The proof of each of these propositions has the same form, namely,

(a) an appropriate graph $G$ is constructed,

(b) it is verified that $\rho(G) \geqslant k$,

(c) it is shown that for each edge $e$ of $G, \rho(G-e) \leqslant k-1$.

It will be convenient in the proof of the theorem to have the following terminology. A colour class of a given colouring of the points of a graph containing just $r$ points is called an $r$-set of the colouring.

THEOREM. Let $k \geqslant 3$ and let $p$ be an even number greater than $2 k$. Then there exists a $k$-critical graph of order $p$.

Proof. As mentioned above, the proof is divided into three stages.

Proposition 1. The theorem holds when

$$
2 k+2 \leqslant p \leqslant 3 k-3 .
$$

Proof of Proposition 1.

(a) Define $h$ by $p=2 k+2 h$. Let $H$ be a graph of order $4 h+3$ whose existence is guaranteed by Lemma 2. Then we construct the graph $G$ of even order $p$ as the join $G=\bar{H}+K_{p-(4 h+3)}$. 
(b) Let us show now that $\rho(G) \geqslant k$. Take an acyclic colouring of $G$ with $\rho(G)$ colours. Denote by $c_{r}$ the number of $r$-sets of the colouring. As $\rho(G)$ is the smallest number of colours in an acyclic colouring of $G$, at most one colour class has only one element, so $c_{1}=0$ or 1 . As $G$ does not have 3 independent points, Lemma 1 implies that every colour class has at most 4 points. Thus

$$
\rho(G)=\sum_{1}^{4} c_{r}
$$

and

$$
p=\sum_{1}^{4} r c_{r}
$$

Furthermore, if a colour class does have 4 points they all belong to $\bar{H}$; if a colour class has 3 points then at least 2 of these points belong to $\bar{H}$. Consequently,

$$
4 c_{4}+2 c_{3} \leqslant 4 h+3 \text {. }
$$

The relations (2) and (1) imply

$$
\begin{aligned}
k & =\frac{1}{4}(2 p-4 h) \leqslant \frac{1}{4}\left\{2 p-\left(4 c_{4}+2 c_{3}-3\right)\right\} \\
& =\frac{1}{4}\left(4 c_{4}+4 c_{3}+4 c_{2}+2 c_{1}+3\right) \\
& =c_{4}+c_{3}+c_{2}+\left(2 c_{1}+3\right) / 4 .
\end{aligned}
$$

As $k$ is an integer and $c_{1}=0$ or 1 ,

$$
k \leqslant \sum_{1}^{4} c_{r}=\rho(G) .
$$

(c) Let $e$ be an edge of $G$. We shall colour $G-e$ acyclically with $k-1$ colours. This will imply that $\rho(G)=k$ and $G$ is $k$-critical.

Suppose first that $e$ joins two points of $\bar{H}$. Then by Lemma 2, H+e contains $K_{3} \cup 2 h K_{2}$ as a spanning subgraph. Colour the points of this $K_{3}$ and a point of $K_{p-(4 h+3)}$ with the first colour. Use each of the next $2 h$ colours to colour the points of a copy of $K_{2}$ (in $K_{3} \cup 2 h K_{2} \subset H+e$ ) and a point of $K_{p-(4 h+3)}$. Divide the remaining $p-(6 h+4)=2 k-4 h-4$ points into $(k-2 h-2) 2$-sets. This gives an acyclic colouring of $G$ with $1+2 h+(k-2 h-2)=k-1$ colours.

Suppose now that $e$ has an endpoint in $K_{p-(4 h+3)}$. Using the facts that $H$ is hamiltonian and $k \geqslant 2 h+3$, one can easily find an acyclic colouring of $G-e$ which has $(2 h+2) 3$-sets and $\{p-3(2 h+2)\} / 2=(k-2 h-3) 2$-sets. Thus $\rho(G-e) \leqslant k-1$, completing the proof of Proposition 1.

PROPOSITION 2. The theorem holds when $3 k-2 \leqslant p \leqslant 4 k-4$.

\section{Proof of Proposition 2.}

(a) Given integers $k \geqslant 3$ and $p$ such that $3 k-2 \leqslant p \leqslant 4 k-4$, it is convenient to introduce an auxiliary parameter $n=p-3 k$. Note then that $-2 \leqslant n \leqslant k-4$. Denote by $L_{2 m}$ the graph obtained from $K_{2 m}$ by removing the edges in an arbitrary 1 -factor.

Construct the graph $G$ by taking the following join:

$$
G=2 K_{2 n+5}+L_{p-4 n-10} \text {. }
$$


(b) Consider an acyclic colouring of $G$ with $\rho(G)$ colours. Then by Lemma 1 every colour class contains at most 4 points. Even more, if 4 points are in a colour class, they are all in the $2 K_{2 n+5}$ part of $G$. Therefore there are at most $(n+2) 4$-sets among the colour classes and the rest are 3-sets or smaller. Counting the number of points in the colour classes, we find that

$$
\begin{aligned}
& \rho(G) \geqslant(n+2)+\frac{3 k+n-4(n+2)}{3} \\
& =(n+2)+(k-n-3+1)=k .
\end{aligned}
$$

(c) Let $e$ be an edge of $G$. Suppose first that $e$ lies in the $2 K_{2 n+5}$. Then we can choose an acyclic colouring of $G$ having a 5 -set colour class in the $2 K_{2 n+5}$. Further, we can form $(n+1) 4$-sets in the $2 K_{2 n+5}$ and the remaining points can be placed into 3-sets. Thus the number of colour classes in this acyclic colouring is just $k-1$.

If $e$ is not in $2 K_{2 n+5}$, then there is an acyclic colouring of $G$ with $(n+2)$ 4-sets of points in the $2 K_{2 n+5}$ and just one more 4-set in $G$. The remaining colour sets can be formed as 3-sets giving in all $k-1$ colour classes in $G-e$. Thus $\rho(G-e) \leqslant k-1$, as required.

Proposition 3. The theorem holds when $p \geqslant 4 k-4$.

Proof of Proposition 3.

(a) Let $p \geqslant 4 k-4$ be even and let $h_{1}, h_{2}$ be natural numbers such that

$$
4 k-8+2 h_{1}+2 h_{2}=p \text {. }
$$

Let the graph $G$ of order $p$ be the join

$$
\left(K_{2 k-6}+C_{2 h_{1}+1}\right) \cup\left(K_{2 k-6}+C_{2 h_{2}+1}\right)+\bar{K}_{2}=\left(H_{1} \cup H_{2}\right)+\bar{K}_{2} .
$$

Before proving that $G$ is $k$-critical, we give another description of $G$.

Let $\widetilde{G}_{i}=K_{2 k-4}+C_{2 h_{l}+1}, i=1,2$, and let $u_{i}, v_{i} \in V\left(K_{2 k-4}\right) \subset V\left(\widetilde{G}_{i}\right), i=1,2$. Put $G_{i}=\widetilde{G}_{i}-e_{i}, i=1,2$, where $e_{i}$ is the edge $u_{i} v_{i}$ of $\widetilde{G}_{i}$. Then $G$ is obtained from $G_{1} \cup G_{2}$ by identifying $u_{1}$ with $u_{2}$ and $v_{1}$ with $v_{2}$. Denote by $u, v \in V(G)$ the points obtained from $u_{i}$ and $v_{i}$. Note that in (3), $V\left(\bar{K}_{2}\right)=\{u, v\}$.

(b) It is easily seen that $\rho\left(\widetilde{G}_{i}\right)=k$ and $\rho\left(H_{i}\right)=k-1$. In fact these are the critical graphs of odd order constructed by Kronk and Mitchem [3].

Suppose $G$ has an acyclic colouring with $k-1$ colours. Then, as $\rho\left(\tilde{G}_{i}\right)=k$, $u$ and $v$ must be of the same colour, say 1 , and there exists a path $P_{i}$ in $\widetilde{G}_{i}-e_{i} \subset G$, connecting $u$ and $v$, whose vertices all have colour 1 . Thus $P_{1} \cup P_{2}$ is a cycle whose vertices are all 1. This contradiction shows that $\rho(G) \geqslant k$.

(c) Let now $e$ be an arbitrary edge of $G$. To complete the proof of the proposition we have to show that $\rho(G-e) \leqslant k-1$. Without loss of generality it suffices to discuss the following two cases.

(1) $e=u w$, where $w \in V\left(H_{1}\right)$. Colour with 1 the vertices $u, v, w$ and another vertex $z$ of $H_{2}$. Choose acyclic colourings of $H_{1}-w$ and $H_{2}-z$ with the same $k-2$ colours, different from 1. Thus we obtain an acyclic colouring of $G$ with $k-1$ colours. 
(2) $e$ is an edge of $H_{1}$. Choose an acyclic colouring of the subgraph $\widetilde{G}_{2}-e_{2}$ of $G$ with $k-1$ colours. In this colouring, $u$ and $v$ must have the same colour, say 1 . As $\rho\left(H_{1}-e_{1}\right)=k-2$, we can find an acyclic colouring of $H_{1}-e$ with $k-2$ new colours. This gives an acyclic colouring of $G$ with $k-1$ colours.

Thus the proof of the theorem is complete. On combining this theorem with the results of [3], we see that for $k \geqslant 3$, there exist $k$-critical graphs of order $p=2 k-1$ and $p \geqslant 2 k+1$ only.

The above proof of the theorem involves the construction of three different families of graphs. This would be simplified by the discovery of a proof using only one construction.

\section{References}

1. G. Chartrand, H. V. Kronk, and C. E. Wall, " The point arboricity of a graph ", Israel J. Math., 6 (1968), 169-175.

2. F. Harary, Graph theory (Addison-Wesley, Reading, Mass., 1969).

3. H. V. Kronk and J. Mitchem, "Critical point-arboritic graphs", J. London Math. Soc. (2), 9 (1975), 459-466.

4. C. St. J. A. Nash-Williams, “Edge-disjoint spanning trees of finite graphs ”, J. London Math. Soc., 36 (1961), 445-450.

Trinity College,

Cambridge University.
The University of Michigan and Wolfson College, Oxford University. 